\title{
El nuevo modelo de Atención Integral en Salud -MIAS- para Colombia. ¿La solución a los problemas del sistema?
}

\section{Germán Alberto Moreno Gomez.}

Profesor titular Facultad de Ciencias de la Salud, Programa de Medicina, Universidad Tecnológica de Pereira. Md. PhD en Salud Pública. MsC en Epidemiologia Clínica, Universidad Tecnológica de Pereira

El gobierno colombiano a través de la resolución 429 de 2016 (1), promulgó la política de Atención Integral en Salud, que pretende generar mejores condiciones de salud para la población a través de la regulación de la intervención sectorial e intersectorial, sustentado en lo dispuesto por un gran volumen de normatividad previa que comprende desde la ley 100 de 1993 y sus posteriores modificaciones incluyendo la ley 1438 de 2011 (2) que pretendía fortalecer y la Atención Primaria en Salud, el Plan decenal de Salud Pública, hasta la de más reciente expedición, la Ley estatutaria 1751, que luego de una gran controversia, consagró por fin la salud como un derecho fundamental. No obstante todos estos desarrollos en materia legislativa, no se han podido resolver los graves problemas por los que atraviesa el sector y que han sido reconocidos por el propio gobierno: fragmentación y desintegración de la atención; baja resolutividad; alta carga de enfermedad; fallos del mercado; incentivos negativos entre los agentes y falla regulatoria, lo que ha conllevado a aumentar la inequidad; corrupción; maltrato al usuario; desorden administrativo; modelo de prestación de servicios de salud morbicéntrico y centrado en los actores; deshumanizado; descontextualizado y sin prevalencia de derechos (2-3).

Surge entonces la Política de Atención integral en Salu y el MIAS como la alternativa para la solución de los problemas y para ello retoma estrategias como la Atención Primaria en Salud con enfoque familiar y comunitario, el cuidado y gestión integral del riesgo y el enfoque diferencial, con lo cual se pretende lograr la articulación y armonización del aseguramiento, la prestación de servicios de salud y el desarrollo de las políticas y programas en salud pública a través de procesos de gestión social y política intersectorial en contexto. Pretende el MIAS garantizar una atención en salud con equidad, centrada en las personas, con estándares de oportunidad, continuidad, integralidad, aceptabilidad y calidad, por medio de procesos de priorización, intervención y arreglos institucionales dirigidos a coordinar las acciones e intervenciones. $(2,4)$

El modelo incluye diez componentes: Se parte de la caracterización de la población, según curso de vida y grupos de riesgo; se definen rutas integrales de atención en salud en promoción y mantenimiento de la salud, grupos de riesgo y eventos específicos de atención; se propone la gestión integral del riesgo en salud, identificando los grupos de riesgo, seguimiento de cohortes, diseñando modelos predictivos, evaluando la efectividad de los servicios y garantizando la interoperabilidad de los sistemas de información; se plantea una delimitación territorial que comprende lo urbano, la alta ruralidad y la población dispersa; las redes integradas de prestadores de servicios de salud con su componente primario y complementario; la definición del rol del asegurador hacia la gestión del riesgo financiero, interacción con otros actores y la gestión de las redes de prestación de servicios; el modelo propone una redefinición del esquema de incentivos, para favorecer la integralidad en la atención y los resultados en salud; un sistema de información centrado en el ciudadano, la familia y la comunidad; el componente de recurso humano propone la formación y armonización para el desarrollo del modelo y mejoramiento de las condiciones laborales. El último componente se dirige al fortalecimiento de la investigación, innovación, y apropiación del conocimiento específicamente en los temas relacionados con rectoría del sistema, sostenibilidad financiera, gestión de recursos, sistemas de información, políticas públicas, acceso uso y calidad del servicio y en Salud Pública. La norma establece un año para el desarrollo de los instrumentos y tres años para implementar el modelo.(5-7)

Si bien es cierto el nuevo modelo trata de incorporar e integrar conceptos y estrategias de probada efectividad y algunas otras novedosas, surgen muchas dudas con respecto a su implementación y efectividad para resolver los problemas planteados, pues en su concepción más profunda y filosófica, están enfrentados dos conceptos sobre la salud: de una parte, la teoría neoliberal utilitarista que considera como fundamentales los derechos civiles y los políticos, que serían los únicos exigibles al estado y no requieren de una norma que los explicite (derechos negativos). En este sentido la salud no es considerada un derecho fundamental, porque según esta concepción, es un derecho de obligación imperfecta que no implica su exigencia. Según la doctrina del liberalismo, la asistencia sanitaria es una prerrogativa social que puede ser solicitada en virtud del principio de beneficencia, al que se accede cuando el individuo lo solicita (3-11).

La contraparte de esta concepción esta soportada en varios autores: Álvarez con base en el trabajo de Amartya Sen del desarrollo como libertad, que considera la salud como un derecho fundamental en la medida en que no basta con explicitarlo (derecho positivo), es necesario trascender el concepto al de oportunidad, entendido como la posibilidad real de acceder a é. Para Gosepath, quien diferencia entre los derechos legales y los morales, (los primeros se materializan a través de la norma y los segundos son inherentes a condición humana), la salud es un derecho moral de la población como parte del respeto igualitario que merece. $(3,8)$ Daniels en su libro "Justice health care" con base en la teoría de la justicia de Rowls, plantea que la salud no se debe fundamentar en la estructura del mercado ya que este no permite la igualdad de oportunidades, lo que va en contravía del principio de justicia y afecta el proyecto de vida de los individuos. Aunado a lo anterior, la relación íntima e insoslayable que tiene la salud con el derecho a la vida, ha hecho que desde el punto de vista jurídico haya llegado a ser exigible. He aquí la gran contraposición que existe entre lo reafirmado por la corte constitucional de la salud como un derecho fundamental y un Sistema de Salud creado bajo la teoría neoliberal. (12-15) 
Este tipo de modelo tiene desarrollos similares en otros países. La evaluación del modelo de atención integral desarrollado en Perú, evidencia aspectos positivos en la implementación, pero se llama la atención en aspectos que no han mejorado o no fueron planteados adecuadamente como integralidad, universalidad, interculturalidad, el enfoque de género, curso de vida, el mayor costo de operación de la estrategia, la confrontación entre una propuesta integral para el primer nivel y un sistema centrado en la atención en los otros niveles de complejidad.(16)

Quedan entonces muchas inquietudes por resolver: ¿Cómo conciliar un modelo regulado por el mercado, con un enfoque patocéntrico con un nuevo modelo basado en APS?, ¿cómo conciliar los intereses del mercado con los intereses de lo público?, ¿cómo conciliar el modelo individualista de la prestación de servicios con un modelo familiar y comunitario?, ¿cómo seguir dejando el desarrollo de la Salud Pública al arbitrio de los aseguradores?, ¿cuándo asumirá el estado su obligación de lo público?, ¿cómo propiciar un modelo participativo y comunitario cuando nunca se ha promovido y educado para esto?, ¿cómo desarrollar un modelo centrado en el usuario cuando no es el actor principal del sistema?

Como se ha demostrado en el pasado, no basta con la expedición de normas que tratan de ajustar un Sistema de Seguridad Social que lo que requiere es una reforma estructural. Parece un decálogo de buenas intenciones que considero, y ojalá esté equivocado, no tiene la fuerza para imponerse sobre el actual sistema.

\section{Referencias}

1. República de Colombia, Ministerio de Salud y de la Protección Social. Resolución 429 de 2016. Política de Atención Integral en Salud

2. República de Colombia, Congreso de la Republica. Ley 100 de 1993. Ley de la Seguridad Social Integral

3. República de Colombia, Congreso de la Republica. Ley 1438 de 2011. Reforma al Sistema General de Seguridad Social en Salud

4. República de Colombia, Ministerio de Salud y de la Protección Social. Plan decenal de Salud Publica 2012-2021. Bogotá 2012.

5. República de Colombia, Congreso de la Republica. Ley estatutaria 1751. Derecho fundamental a la Salud. Bogotá. Febrero de 2015

6. República de Colombia, Ministerio de Salud y de la Protección Social. Lineamientos generales para el desarrollo del modelo integral en atención en salud para el sistema general de seguridad social en salud.Bogotá. Diciembre de 2014

7. Castaño Alvares LS. El derecho a la salud en Colombia: una propuesta para su fundamentación moral. Rev Panam Salud Publica/Pan Am J Public Health 18(2), 2005.

8. Gracia D. Fundamentos de bioética. Madrid: Eudema; 1989

9. Sen A. Desarrollo y libertad. Santa Fe de Bogotá: Planeta; 2000

10. Gosepath S. Consideraciones sobre las fundamentaciones de los derechos humanos sociales. En: Alonso MA, Giraldo J, eds. Ciudadanía y derechos humanos sociales. Medellín: Escuela Nacional Sindical; 2001. Pp.15-58

11. Daniels N. Justice health care. Cambridge (U.K.): Cambridge University Press; 1985

12. Rawls J. Teoría de la justicia. México, D.F.: Fondo de Cultura Económica; 1997

13. Jara Navarro MI. Ética de la salud como ética de la vida. Rev Gerencia y Políticas de Salud. 2001: 1

14. Naranjo J, Delgado A, Rodríguez R, Sánchez Y. Consideraciones sobre el modelo de atención integral de salud del Ecuador. Revista Cubana de Medicina General Integral. 2014; 30(3)

15. República de Guatemala. Gobierno de la República. Ministerio de Salud Pública. Modelo de Atención Integral en Salud.

16. Feo O, Tobar K. Informe de evaluación "Modelo integral e incluyente bajo un concepto de derecho a la Salud" Peru. 2014 